\title{
Kernos
}

Revue internationale et pluridisciplinaire de religion grecque antique

$4 \mid 1991$

Varia

\section{Nouveaux points de vue sur la mentalité religieuse des Romains}

Gerhard Radke

\section{OpenEdition}

\section{Journals}

Édition électronique

URL : http://journals.openedition.org/kernos/282

DOI : $10.4000 /$ kernos.282

ISSN : 2034-7871

\section{Éditeur}

Centre international d'étude de la religion grecque antique

\section{Édition imprimée}

Date de publication : 1 janvier 1991

Pagination : 31-46

ISSN : 0776-3824

\section{Référence électronique}

Gerhard Radke, "Nouveaux points de vue sur la mentalité religieuse des Romains », Kernos [En ligne], 4 | 1991, mis en ligne le 11 mars 2011, consulté le 22 avril 2019. URL : http://journals.openedition.org/ kernos/282 ; DOI : 10.4000/kernos.282 
Kernos, 4 (1991), p. 31-46.

\section{NOUVEAUX POINTS DE VUE SUR LA MENTALITÉ RELIGIEUSE DES ROMAINS *}

Les Romains se considéraient comme le peuple le plus religieux du monde ${ }^{1}$, et l'historien grec Polybe ${ }^{2}$ reconnut, dans leur intense crainte des dieux, l'une des causes de leur supériorité établie sur les autres peuples. L'historien d'aujourd'hui s'interroge cependant encore sur la nature de cette piété. Il doit étudier les ressorts de la vénération divine; il doit identifier ce que les Romains attendaient de leurs dieux; il doit comprendre par quels moyens ils cherchaient à gagner une influence sur l'action divine; il doit préciser l'originalité et la particularité des êtres divins; il doit enfin décrire la mentalité religieuse, c'est-à-dire comment, dans l'esprit des Romains, s'est formée l'image de leurs divinités.

\section{Les rapports entre les dieux}

Le domaine envisagé est cependant limité par des restrictions mentales, dont la plus importante est l'incapacité d'imaginer entre les dieux des relations sexuelles ou des rapports de parenté, comme la mythologie grecque en fournit l'exemple. Pour illustrer cette affirmation, on peut prendre le cas des relations entre Jupiter et Junon, dont la ressemblance avec le couple formé par Zeus et Héra dans la mythologie grecque n'est qu'apparente. On n'y trouverait aucune correspondance fondamentale. La Triade capitoline à Rome, qui insère Jupiter entre deux déesses, Junon et Minerve, interdit déjà par cette construction l'idée d'un mariage du dieu avec l'une de ses deux compagnes : le Jupiter romain, malgré toutes les liaisons mythiques rapportées sur son pendant grec Zeus, n'était guère bigame. Il était indubitablement célibataire et l'idée d'une relation sexuelle de ce dieu romain reste inconnue! Pourtant, on doit concéder qu'il y a une analogie étymologique et même fonctionnelle entre Jupiter et Zeus, tous deux étant les dieux du ciel clair. Par contre, Junon ne peut pas être identifiée à Héra : d'après le sens de son nom, Junon était originairement la représentante des femmes en âge de concevoir et, garante ainsi de l'avenir productif, elle

* Conférence donnée au Collège de France, Paris, en octobre 1988.

1 Salluste, Catil., 12, 3; Aulu Gelle, II, 28, 2.

2 PolyBe, VI, 56, 6 sq. 
se voyait allouer les fonction de gardienne de la ville afin d'en assurer la survie. Hors de la triade capitoline, on ne trouve aucun culte archaïque qui associe Jupiter et Junon.

La conception romaine des Dioscures confirme l'ignorance de relations de parenté entre les dieux. Alors que les Péligniens et les Marses, peuples italiques, les appelaient Iovios puclos ${ }^{3}$ et que les Étrusques, par les mots de tinas cliniiaras $^{4}$, traduisaient de même exactement leur nom grec de «fils de Zeus», à Lavinium, tout différemment, une inscription latine très ancienne s'adresse aux Castorei Podlouqueique qurois $^{5}$ sans mentionner le nom de leur père ou le degré de leur parenté. Ces dieux n'étaient que des jeunes hommes, des iuvenes. Les Romains, de leur côté, les vénéraient même sous le nom de l'un d'entre eux, en appelant aedes Castoris $^{6}$ le temple qui leur était dédié, parce que l'idée de dieux jumeaux à Rome n'était pas connue et la descendance d'un dieu inconcevable. Comme le démontre cet exemple, l'aversion des Romains à l'égard de l'idée qu'il puisse exister des rapports de parenté entre les divinités était si forte qu'elle réussissait même à effacer les relations qui existaient déjà entre les dieux étrangers avant leur arrivée en Italie.

On le remarquera, par un autre exemple, en observant la dénomination divine : lorsque la Fortuna Iovis filia Primigenia de Préneste, la fille de Jupiter dans sa patrie, reçut un culte à Rome en 194 av. J.-C., on supprima l'allusion à sa filiation jupitérienne et on l'appela dans son temple romain Fortuna publica populi Romani Quiritium Primigenia 7 .

La coutume de s'adresser à une divinité en utilisant le terme de pater ("père») ne correspond pas à une figuration de la paternité, mais exprime le respect, l'affection aussi envers le dieu ainsi dénommé. Cependant une déesse appelée mater («mère») joue un autre rôle, mais ni Mater Matuta ni Vesta Mater ne furent comprises comme la mère

3 E. VeTter, Handbuch der italischen Dialekte, I, Heidelberg, 1950, n 202, 224.

4 M. PALLoTTINo, $T L E^{2} \mathrm{n}^{\circ} 156$.

5 A. DEGRASI, ILLRP $2, \mathrm{n}^{\circ} 1271 \mathrm{a}$.

6 Plaute, Curculio, 481; CIC., Verrines, II, 1, 129 sq.; Tite Live, II, 20, 12; FesTUS, p. 362, 29 Lindsay; CIL, I2, 586. Cf. G. RADKE, Zur Entwicklung der Gottesvorstellung und der Gottesverehrung in Rom, Darmstadt, Wissenchaftliche Buchgesellschaft, 1987, p. 179 sq.

7 Tite Live, XXIX, 36, 8; XXXIV, 53, 5. Pour le 25 mai, cf. Fasti Esquilini, CIL, I2, p. 211 (= A. DEgRASSI, Inscr. Ital. XIII 2, p. 87) Fortunae public in colle; Fasti Caeretani, CIL , I' , p. 213 (= I, I, p. 67) Fortunae PPRQ in colle Quirin; Fasti Venusini, $C I L, \mathrm{I}^{2}$, p. 221 (= I, I, p. 56-57) Fortun Prim in col. 
d'autres divinités : sous l'épiclèse mater, elles remplirent leur fonction spécifique; que l'une ait incarné le modèle des mères humaines, l'autre la terre d'après l'interprétation de Varron ${ }^{8}$, resta sans importance pour l'explication sommaire.

Il y eut d'abord à Rome une époque au cours de laquelle on ne savait pas encore préciser le sexe d'une divinité et l'on se vit obligé de recourir au neutre pour la désigner, comme cela s'est maintenu dans les noms de Cérès et de Vénus, formés par un suffixe originairement neutre. Par la suite, on vit apparaître une autre mentalité religieuse, consciente de l'existence des déesses à côté de celle des dieux, mais on ne sut pas encore à quel sexe appartenait la divinité invoquée à une occasion particulière; on s'adressait alors sive deo sive divae" ("soit à un dieu, soit à une déesse») pour ne pas être embarrassé par l'ignorance du sexe divin. Lorsqu'on voulait le préciser, on divisait l'unité divine originaire en deux moitiés; l'une recevait un nom masculin, tels que Liber, Faunus, Cacus; l'autre, un nom féminin comme Libera, Fauna, Caca. Mais aucune relation sexuelle ne liait ces deux moitiés. Car les histoires sur Faunus et Fauna ne sont que la traduction des romans grecs ${ }^{10}$, et le partenaire de la Caca romaine n'était qu'un monstre à la gueule crachant $d u$ feu dans la description virgilienne ${ }^{11}$. Liber et Libera, dont la fête, les Liberalia, est plus ancienne que le culte de la triade aventine $\mathrm{e}^{12}$, ne sont pas liés par une soi-disant parenté, issue de la descendance de Cérès, comme Cicéron le suppose ${ }^{13}$, séduit par le récit d'un guide ingénieux, mais ils représentaient l'expression de fonctions qui renvoient Liber aux hommes pour les délivrer de leur sperme et sa partenaire Libera aux femmes avec un but analogue ${ }^{14}$.

L'assertion de Varron selon laquelle les Romains ne recoururent pas à des idoles pour représenter les divinités pendant plus de 170 ans après la fondation de Rome ${ }^{15}$, illustre cette conception. En considérant

8 VARRON, fr. 281 Cardauns; cf. fr. 268.

9 A. DEGRASSI, ILLRP², n ${ }^{\circ} 291$ sq.; CATON, Agr., p. 139; AULU Gelle, II, $28,3$.

10 Cf. G. RADKE, Die Götter Altitaliens, Münster, 1979², p. 121.

11 VIRG., En., VIII, p. 194-303.

12 Pour le 17 mars (Liberalia), cf. Fasti Caeretani, CIL, I2, p. 212 (= I, I, p. 66) Libero Lib; pour le 19 avril (Cerialia), cf. Fasti Esquilini, CIL, I2 , p. 210 (= I, I, p. 86) Cerei Libero [Liberae].

13 CrC., Div., II, 85.

14 Augustin, Civitas Dei, VI, 9.

15 VARRON, fr. in AUGUSTIN, op. cit., IV, 31. 
que la divinité était avant tout une puissance agissante, mue par les charges spécifiques qui lui étaient attribuées, les Romains n'avaient pas de raison de lui donner une apparence humaine, réductrice de la personnalité unique de la divinité. Ainsi la lance ${ }^{16}$ dressée dans le sanctuaire ne représente pas plus le dieu Mars que la reproduction en bois ou en argile d'un membre viril ne représente le dieu Liber ${ }^{17}$. Ces symboles ne sauraient traduire à eux seuls la complexité de l'essence de ces divinités selon la pensée religieuse des Romains. Toutefois la civilisation grecque exerça finalement une influence prédominante sur la représentation des divinités chez les Romains : par exemple, une femme portant des enfants sur ses genoux traduit clairement l'idée d'une déesse mère ${ }^{18}$. D'après ce que l'on sait du culte romain, les premières images des dieux romains à modèle humain ont été Jupiter ${ }^{19}$, Diane aventine après la visite des Phocéens à Rome ${ }^{20}$ et également Fortune. La statue de cette dernière passait aux yeux de ceux qui la vénéraient pour le roi Servius Tullius parce que l'image fut façonnée à partir d'un modèle humain ${ }^{21}$, plus mâle que féminin.

Orcevia, une femme de Préneste, qui remercia Fortuna Iovis filia Primigenia pour ses couches ${ }^{22}$, constitue, au début du IIIe siècle av. J.C., l'un des premiers exemples documentés qui représentent des relations de parenté entre des divinités. Cependant c'était une conception prénestine, non romaine. Mais si les Romains ont donné à certains dieux une forme humaine, ils les maintinrent au rang de divinité et ne leur attribuèrent pas de faiblesses humaines comme l'amour, la répulsion, la jalousie ou l'avidité qui caractérisent les dieux d'Homère.

16 VARRON, fr. 234 Cardauns.

17 VARRON, fr. 262 Cardauns.

18 CIC., Div., II, 85; cf. les statues des déesses assises à Satricum : Della SETA, Museo di Villa Giulia, 1, p. 303 sq.

19 Puine, Nat. Hist., XXXV, 157.

20 Strabon, IV, p. 179 sq. Cf. F. Altheim, Die griechischen Götter im alten Rom, Gießen, 1930 (RGVV, 22, 1), p. 136 : лótvı

21 Pline, Nat. Hist., VIII, p. 197; cf. J. Champeaux, Le culte de la Fortune à Rome et dans le monde romain, Rome, 1982 (Coll. Ecole Française à Rome, 64), p. 274 sq.

$22 C I L, \mathrm{I}^{2}$, p. $60=I L L R P^{2}, \mathrm{n}^{\circ} 101$. 


\section{Noms et activités des dieux}

Ce sont les dénominations des dieux dans le culte qui donnent le plus facilement la clé de leur nature et de leurs attribution. Le nom décrit la fonction d'un dieu; il fait comprendre son caractère. Cette assertion de Varron ${ }^{23}$, on ne peut que la confirmer et y ajouter que c'étaient naturellement les Romains qui exprimaient à travers les noms qu'ils donnaient à leurs divinités ce qu'ils en avaient déjà reçu ou ce qu'ils en attendaient encore et en obtiendraient par leurs prières et leurs intercessions.

Le rôle décisif fut joué ici par l'usage des suffixes qui désignent un rapport entre la chose nommée et le verbe auquel le suffixe fut ajouté 24 . Il y en a beaucoup, parmi lesquels le suffixe -io, au féminin -ia, est le plus fréquent; il marque la dépendance ${ }^{25}$ : Genius, par exemple, est attaché à la procréation, Seia s'intéresse aux semailles, Aius a la fonction de la parole, Maia possède le pouvoir d'agrandir et Virbius a le don de redresser.

Je me contente par la suite de présenter le suffixe $-t a$ dans la fonction d'un participe parfait actif qui indique l'exécution d'une action : parta quae peperit ${ }^{26}$; une femme qui a accouché est appelée parta. L'unique exemple dont je ferai usage est l'épiclèse "Matuta» qui contient le terme *matu- «bon» et qualifie une déesse qui a permis un heureux dénouement. La Pales Pastoria, après la victoire que les Romains remportèrent grâce à son aide, fut appelée Pales Matuta ${ }^{27}$. Iuno Sospita, après l'accomplissement d'une prière qui lui avait été adressée, reçut un temple à Rome; dans le culte nouveau, la déesse fut désignée par le nom de "Iuno Matuta»28. Enfin, "Mater Matuta" représente la fonction d'une mère après l'accouchement ${ }^{29}$. Un des cultes les plus antiques de Rome est celui de Vesta dans son sanctuaire rond et non inauguré, élevé à l'emplacement d'un cimetière préhistorique. Selon la tradition, la

23 VARRON, fr. 87 Cardauns.

24 Cf. G. RADKE, Die Götter Altialiens, Münster, 19792, p. 12 sq. ID., Das Wirken der römischen Götter, in Gymnasium, 77 (1970), p. 29.

25 Gĕnius <* gnn-iðs, Seia < * sě-s -iā, Aius < *ag-iðs, Maia <*magh-iāa,Virbius <

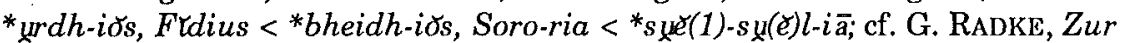
Entwicklung der Gottesvorstellung und der Gottesverherung in Rom, Darmstadt, 1987, p. 182 sq.

26 Columelle, VII, 4, 3; cf. SERV. auct. Én., VIII, 630. Schol. Bern. Eclog., I, 50.

27 Florus, I, 15; Schol. Bern. Georg., III, 1; Schol. Veron. Georg., III,1

28 TITE Live, XXXII, 30, 10; XXXIV, 53,3.

29 Ovide, Fastes, VI, p. 479. 
déesse fait garder le feu perpétuel par ses prêtresses, les vestales, dont la chasteté garantit la pérennité. Mais il est difficile de trouver une relation entre ces deux tâches, le feu et la virginité. Plus énigmatique encore est le nom de la déesse : les théories avancées jusqu'à présent le font dériver des racines qui signifient "être domicilié" ou "manger" ou "brûler" ou "fourneau" ou "bon", mais elles n'ont réussi à élucider ni la signification du nom de Vesta ni la fonction de son culte. Or celui-ci trouve son explication dans le rôle originel des vestales : ce rôle est analogue à celui des jeunes filles, qui, dans la mythologie grecque, furent précipitées dans la mer du haut d'un rocher parce qu'elles avaient eu des relations avec un homme contre le gré de leurs parents ${ }^{30}$. À cause de ce faux pas, elles ont abandonné leur famille et se sont exclues de la communauté. Ce phénomène social se fonde sur des coutumes méditerranéennes prégrecques.

La finalité de cette mise à mort n'est pas de punir; mais on tient la pécheresse pour un élément dangereux qui trouble la paix de la communauté; aussi veut-on l'éliminer ou l'éloigner du pays afin de ne pas être souillé par son impureté. Dans d'autres versions, la lapidation ou l'ensevelissement de la coupable vivante, comme le subissaient les vestales incestueuses à Rome, remplaçait la chute du haut d'un rocher. Pour éliminer une souillure ou pour repousser un danger menaçant, le clergé avait, par avance, à sa disposition un stock permanent de victimes désignées; il organisa ainsi le service des vestales, délivrées de la puissance paternelle sans perdre toutefois leur propre identité civile $^{31}$, mais obligées de garder une chasteté absolue sous la protection de Vesta. En tout cela il y a une analogie avec le service historique des jeunes Locriennes à Ilion à cause du forfait mythique d'Ajax ${ }^{32}$.

Dans ces conditions, l'opinion de Hildebrecht Hommel ${ }^{33}$ parait être fondée qui reconnaît un aspect phallique au culte de Vesta, ainsi qu'en témoigne le fascinus, le membre viril vénéré par les vestales ${ }^{34}$. La fonction religieuse spécifique de la déesse serait ainsi probablement la fécondation. De même, à mon avis, l'étymologie du nom de Vesta concorderait avec cette interprétation : je le dérive d'une racine *uers-

30 Cf. G. RADKe, op. cit., p. 263; Schol. LyCoPhron, 1141 se sert du mot $\dot{\alpha} \theta \varepsilon \mu \imath \tau о \mu \imath \xi \hat{\prime} \alpha$.

31 AULU Gelle, I, 12,9; GAIUS, Institutions, I, p. 130.

32 Serv. auct. En., I, 41; APOLLOD., Epitome, V, p. 20 sq.; PLuT., Mor., 55c-d.

33 H. Hommel, Vesta und die frührömische Religion, in ANRW, I, 2, Berlin, 1975, p. 419 sq.

34 PLINE, Nat. Hist., XXVIII, p. 39. 
qui signifie "arroser, féconder" 35 . L'analyse linguistique du nom de la déesse condamne l'explication comme nomen actionis et permet uniquement la fonction d'un participe parfait passif. Ainsi le nom de Vesta signifierait *Versta >Vesta «la fécondée», à quoi correspond la dénomination Vesta Mater. La déesse serait alors apparemment la projection des jeunes filles qui ont mis au monde un enfant contre la volonté de leurs parents, c'est-à-dire un enfant de vierge ${ }^{36}$.

Mais comme aucun culte romain n'est motivé par un événement mythique et comme l'analogie des jeunes filles précipitées du haut d'un rocher ne tend qu'à fournir un argument pour cette mise à mort conforme aux mœurs sévères d'une société méditerranéenne, la déesse romaine, pleine de sollicitude pour ces jeunes filles, donne à cet usage une représentation rituelle. Aussi le culte de Vesta comprend-il un acte procréateur, mais celui-ci évite une contamination avec les fautes humaines, parce qu'il concerne une fonction centrale de la nature : c'est la terre même dont la fécondité est marquée par le nom de Vesta, d'après une observation qui remonte à Varron ${ }^{37}$. Puisque la fécondité agraire et la fécondité animale ou humaine ont une valeur identique dans tous les cultes, la chasteté des vierges au service de la déesse et la vénération du membre viril dans son culte correspondent à la même idée de protection et d'incarnation de la fécondité par Vesta. En outre le fait que sa fête, les Vestalia, célébrée le 9 juin, soit suivie des Matralia le 11 juin confirmerait à mon avis cette thèse.

Par conséquent je crois que les Vestalia célèbrent la fécondité de la terre; c'est la fête de la moisson. En Italie, on récolte le froment avant le solstice d'été ${ }^{38}$ et l'épeautre encore plus tôt. Dans la deuxième semaine du mois de mai, les épis d'épeautre, c'est-à-dire de far, espèce de blé cultivée depuis une époque reculée, ne sont pas encore mûrs, mais déjà assez grands pour être tranchés et utilisés dans la fabrication de la mola salsa par les vestales ${ }^{39}$. On peut en conclure que c'était la moisson du far, du blé le plus ancien, nourriture préhistorique des Romains, qui est à l'origine de la fête.

35 Cf. G. RADKE, op. cit., p. 284 sq.

36 Cf. G. RADKe, L'enfant de la vierge, in La mythologie, clef de lecture du monde classique, Tours, 1986 (Caesarodunum, XXI bis), p. 1 sq.

37 VARron, fr. 281 Cardauns; cf. fr. 268; Ovide, Fastes, VI, p. 276; Festus, p. 320, 14 Lindsay.

38 VARron, Rer. Rust., I, 32, 1.

39 PLINE, Nat. Hist., XVIII, 7; SERV. auct. Éclog., VIII, 82. 
De même qu'à Rome la mère après ses couches doit passez par une période de purification, de même, après cette fête de la moisson, le temple de Vesta était nettoyé pendant neuf jours ${ }^{40}$. On ne peut ignorer cette analogie. À la suite des légendes latines racontant la procréation par une étincelle ou une flammèche du foyer ou par un membre viril s'érigeant du feu ${ }^{41}$, il faut établir une liaison fonctionnelle entre la procréation et le feu du foyer dont la perpétuité correspond à l'éternel retour de la croissance végétale. Ce lien entre le feu et la fécondité explique la double tâche de Vesta.

\section{Jupiter}

Je reviens encore une fois à Jupiter, mais sous un point de vue différent : il ne fait aucun doute que la formation du nom de ce dieu éclaire la nature de sa divinité. À l'époque archaïque on composa ce nom à partir de deux mots, l'un désignant le ciel clair, l'autre le père. En outre il est généralement admis qu'à l'origine Jupiter fut formé sur un vocatif. Le nominatif composé avec pater ne se serait formé en latin que par la suite, sur le modèle vocatif. Le vocatif, forme grammaticale de l'interpellation directe, a pu être utilisé dans le cas de Jupiter comme nominatif, cas habituellement plus affirmatif et décoloré. Cela reflète le caractère de la foi que les Romains avaient envers leur dieu. Cette transformation grammaticale peut être considérée comme tout à fait exceptionnelle; elle est l'exemple unique, dans l'histoire des religions européennes, d'un nominatif tirant son origine d'un vocatif. On pourra donc dire que le Jupiter romain est un dieu pour qui l'invocation est partie intégrante de l'existence ${ }^{42}$, qualité divine exactement appropriée pour établir une suprématie particulière.

En effet, cette représentation imaginaire du dieu s'est transformée vers la fin du VIe siècle av. J.-C. La fondation du temple capitolin fut un acte de portée à la fois religieuse et politique. Les Romains découvrirent la conception d'une divinité dont l'unique fonction était l'ordre de l'État. Dans la formule juridique de la dénomination de Iuppiter Optimus Maximus, qui signifie un dieu libre de toute servitude, libre de toute dépendance des règles rituelles, la jeune république sut affirmer l'unité des tribus autour de la ville et exprimer en même temps une nouvelle forme de nation grâce à un culte neuf, dont les divinités reflètent alors la situation politique. La triade capitoline, Jupiter, Junon

40 Philocale, CIL., I' 2 , p. 266.

41 Cf. Caeculus, Servius Tullius et les petits-enfants du Tarchetios.

42 Cf. G. RADKE, op. cit., p. 238. 
et Minerve, triade non étrusque, mais typiquement romaine, est en effet l'expression d'une idée nationale née de la volonté politique de la res publica romana. La République voulut ainsi affirmer son autonomie, à l'image d'un dieu souverain ${ }^{43}$.

\section{L'influence étrusque}

On a toujours surestimé l'influence des Étrusques dans la vie culturelle et cultuelle de Rome. Il faut corriger cette façon de penser. Il y a plus de vingt ans, j'ai démontré que la forme écrite du nom Apollon qui se trouve dans les inscriptions étrusques dès le Ve siècle av. J.-C., apulu, malgré l'habitude normale de garder la désinence originale des noms importés, comme charun pour le grec Charon, la forme apulu du nom d'Apollon dérive directement de la dénomination romaine Apollo sans un $-n$ à la fin. Le latin est en effet l'unique langue italique dans laquelle le nominatif singulier des termes comme ordo, ordinis, homo, hominis a perdu la terminaison $-n$. Cela veut dire que le nom grec d'Apollon fut changé à Rome en Apollo sans - $n$ final et transmis après cette assimilation latine comme apulu en Étrurie ${ }^{44}$. Les étruscologues m'ont donné raison sur ce point 45 .

Le cas le plus important qui contredit nettement l'idée d'une origine étrusque concerne le dieu Saturne : je suis convaincu que le nom de Saturnus - originairement écrit Saeturnus avec diphtongue - se compose d'une racine qui signifie "semer" et d'un infixe causatif - je- dont l'insertion avait produit la forme *saieturno-, Saeturno- et par conséquent allongé la première syllabe; du fait de sa fonction, cet infixe indique que la structure du nom signifie "faire semer, provoquer à semer». Sans discuter ici la raison de la désinence -turno-, on peut en déduire que Saturne a reçu son nom des Romains parce qu'il leur avait appris l'art des semailles ${ }^{46}$. En effet sa fête fut fixée dans le calendrier au moment où l'ensemencement hivernal est terminé, soit quinze jours avant la journée la plus brève, la bruma. Cette date suppose qu'au temps de l'organisation du calendrier, la bruma n'était pas le 24 décembre comme dans le dernier temps av. J.-C., mais six jours plus tard, le 30

43 Cf. G. RADKE, I.O.M., dieu libre de toute servitude, in $R D, 64$ (1986), p. 1 sq.

44 Cf. G. RADKE, Die Götter Altitaliens, Münster, 19792, p. 53.

45 A.J. Pfiffig, Religio Etrusca, Graz, 1975, p. 251; E. Simon, Apollo in Rom, in $J D A I, 93$ (1978), p. 206, n. 30; H. RIX, Rapporti onomastici fra il panteon etrusco e quello romano, in Gli Etruschi e Roma, Rome, 1981, p. 126, n. 79. Quant à Prosperpine, cf. G. RADKE, op. cit., p. 41 sq. 
décembre selon l'ère julienne. Cela nous replace vers 700 av. J.-C., c'est-à-dire sous le règne de Numa Pompilius, dans l'hypothèse où les points fixes d'année reculent d'un jour tous les 128 ans ${ }^{47}$. Par conséquent l'explication varronienne qui lie le nom du dieu à satus «les semailles», terme qui comporte une voyelle brève à la première syllabe, n'est pas totalement erronée; mais elle ferme seulement un œil sur le renseignement précis, tiré de la structure du nom de Saturnus avec une voyelle longue dans la première syllabe ${ }^{48}$ : l'étymologie prouve que le dieu avait pour fonction de faire semer, d'inciter à semer et d'indiquer aux Romains le moment propice pour y procéder. La date reconstituée confirme cette interprétation 49 .

Même si Varron ${ }^{50}$ pouvait interpréter le nom de quelques dieux, on ne saurait prétendre que les Romains au temps de Cicéron comprenaient encore leur signification. Mais l'ignorance d'une époque récente ne donne pas le droit de renoncer à l'aide qu'offre la langue d'autrefois. Cependant il convient d'admettre que la représentation des êtres divins avait perdu à la longue de sa valeur; la discipline du culte, en l'emportant sur la pensée religieuse, ne permit plus à l'imagination une représentation individuelle de la divinité.

\section{Les sacrifices}

Pour l'homme qui s'adresse au dieu, la connaissance du nom divin revêt plus d'importance que la connaissance du sexe. C'est bien compréhensible. La dénomination exprime la fonction divine : il faut utiliser avec précision ce nom transparent, ce nom qui révèle ce que la divinité peut accomplir. La connaissance du nom confère au rapport une signification magique, oblige la divinité à remplir l'action exprimée à travers son nom et par cela déjà promise; elle confère à l'homme le pouvoir de forcer la divinité à apparaître à son appel. Quand je parle du diable, il arrive. Naturellement, la présence d'un dieu est un événement effroyable, de sorte que l'homme lève les mains spontanément pour détourner cet être inconnu surnaturel, dont il ne sait s'il veut aider ou nuire; le geste de prière à la fois spontané et magique d'étendre les paumes vers le ciel, n'est qu'une défense.

47 E. NoRden, Die Geburt des Kindes, Teubner, Leipzig, 1924, p. 38.

48 VARRON, Ling. Lat., V, 64.

49 Cf. G. Wissowa, Religion und Kultus der Römer, München, 1912², p. 204.

50 VARRON, fr. 87 Cardauns; par exemple Altor, Rusor (fr. 266), Ceres (Ling. lat. V, 64), Diana (Ling. lat., V, 68), Genius (fr. 248), Liber (fr. 93), Prosperpina (Ling. lat., V, 68), Saturnus (fr. 239/241), Venilia, Salacia (fr. 257), Vesta (fr. 268). 
Lorsque l'homme entre en communication avec le dieu, il peut alors prier pour l'accomplissement de ses désirs. Mais cette prière est un acte de commerce. Dans son vœu, l'homme promet aussi un service à la divinité. Par conséquent, l'interprétation du terme religio par le verbe religare, "lier» ${ }^{51}$, ne correspond pas à la réalité. La pensée religieuse exige au contraire une observation précise des règles du contrat fait avec le dieu, si l'on suit l'explication proposée par Cicéron ${ }^{52}$ qui rattache religio à un verbe religere, "observer", en décrivant exactement les relations entre les deux mondes. Ainsi le texte d'une prière, surtout d'une prière prononcée par un prêtre au nom de la communauté, est fixé mot à mot; le texte, par exemple, du chant arval a été conservé littéralement de l'époque archaïque jusqu'à une inscription de l'an 218 ap. J.-C. ${ }^{53}$, que l'on peut lire encore aujourd'hui. Une différence dans la récitation, si petite soit-elle, rendait l'acte cultuel inefficace et contraignait à répéter la cérémonie.

De telles motivations et de telles contraintes valent aussi pour les sacrifices au temps des Romains. Le terme sacrificium signifie "acte par lequel quelque chose devient sacré», c'est-à-dire cédé aux dieux. Pourtant le sacrifice n'est pas un cadeau donné à une divinité. Le geste résulte presque exclusivement de la peur du danger que représente pour l'homme la consommation d'aliments non encore goûtés. Les Romains ne mangeaient aucun fruit et ne buvaient pas de vin nouveau avant qu'un prêtre n'ait offert un échantillon aux dieux ${ }^{54}$. Cela valait aussi pour le praemetium ${ }^{55}$ pendant la moisson du blé, pour la taille première de la vigne ${ }^{56}$, pour la sacrima ${ }^{57}$, pour les fèves nouvelles ${ }^{58}$, pour le vin puisé d'un tonneau nouveau ${ }^{59}$, pour les épis premiers fleurissants ${ }^{60}$ : on confiait aux dieux la responsabilité de goûter les premiers produits de la terre, épargnant ainsi à l'homme tout risque d'empoisonnement.

51 LACTANCE, Inst. div., IV, 28 p. 3 sq. SERV., En., VIII, 349.

52 CIC., Nature des dieux, II, 72; Part. orat., p. 78; cf. fr. trag. in AUlu Gelle., IV, 9 , 1 : religentem esse oportet, religiosus ne fuas.

$53 C I L, \mathrm{I}^{2}, 2=I L L R P^{2}, \mathrm{n}^{\circ} 4$.

54 PuINe, Nat. Hist., XVIII, 8.

55 PAUL. Festus, p. 267, 1; 423, 2 Lindsay.

56 VARRON, Ling. lat. , VI, 16.

57 Paul. Festus, p. 423, 1 Lindsay.

58 PAUL. FEstus, p. 344, 3; p. 345, 1 Lindsay.

59 Paul. Festus, p. 57, 16 sq. Lindsay.

60 Paul. Festus, p. 81, 5 Lindsay; Augustrn, Civitas Dei, IV, 8. 
Les mêmes arguments justifient aussi les sacrifices d'animaux. Kurt Latte ${ }^{61}$ a prétendu que «chaque sacrifice romain a le caractère d'un repas qu'on offre aux dieux et auquel les hommes participent aussi». Cela décrit à mon avis exactement les fondements du sacrifice romain, que Jean-Pierre Vernant ${ }^{62}$ précise ainsi : «Sacrifier, c'est fondamentalement tuer pour manger». L'homme tue un animal pour le manger. Après la mise à mort, il examine les entrailles pour s'assurer, par leur état, de la qualité irréprochable de la bête. Pendant ces rites, il s'adresse à une divinité, lui montre la victime et, par la formule macte esto, prie le dieu de se sentir honoré par cette inspection offerte ${ }^{63}$. Puis il cède aux dieux les parts non mangeables, mais aussi les magmenta, c'est-à-dire des petits morceaux de bonne viande, pour étendre l'efficacité et la valeur de l'examen divin, non seulement aux parties non comestibles et brûlées sur l'autel, mais aussi à la viande qu'il porte au dehors du sanctuaire et qu'il désacralise ainsi. On est ainsi assuré que la viande est sans défaut et bien apte à la consommation. Cet acte sacrificiel, comme Caton l'a décrit, doit être compris comme une mesure d'hygiène : l'examen sous les yeux et avec l'aide de la divinité épargne aux consommateurs des intoxications, tandis que la combustion des parties non comestibles écarte les épidémies.

À l'occasion des grandes fêtes, on sacrifiait des taureaux aux dieux et des vaches aux déesses, mais ces hécatombes tendaient à nourrir non pas les divinités, mais la communauté. Malgré cet objectif tout à fait quotidien et banal, on a observé les prescriptions rituelles parce que l'hygiène en dépendait obligatoirement. Les règles cultuelles servaient à protéger la santé de la population et à la garder des maladies épidémiques. Ainsi peut-on éviter toutes les spéculations qui ont obscurci l'histoire des religions. La pensée religieuse s'appuie sur les nécessités les plus simples et les plus réelles de la vie romaine. La croyance en la justice divine aide celui qui la mérite, parce qu'il a suivi les lois religieuses fondées sur les exigences fondamentales de la vie humaine.

\section{Dieux étrangers}

$\mathrm{Ni}$ le culte grec ni le culte romain ne pratiquaient le prosélytisme; ils ne font pas de propagande pour leurs propres dieux auprès des nations étrangères. De ce fait, il est difficile de connaitre les motifs ou les

61 K. LATTE, Römische Religionsgeschichte, München, 1960, p. 391.

62 J.P. VERNANT, Théorie générale de sacrifice..., in Le sacrifice dans l'antiquité, Vandœuvre-Genève, 1981 (Entretiens sur l'Antiquité classique, 27), p. 18, 26.

63 Caton, Agr., p. 132, 134, 139, 141. 
formes sous lesquelles les divinités grecques sont entrées dans la religion romaine. Personnellement, j'ai l'impression que les premiers contacts des Romains avec les dieux étrangers furent pour eux comme une aventure émouvante. Je répète pour cela un fait déjà mentionné. Il s'agit de dieux dont la renommée s'était répandue en Italie comme une inondation, après la confirmation de leur aide active et efficace lors de la bataille du fleuve Sagra après 530 av. J.-C. Les Locriens du moins, bénéficiaires de cette présence divine, étaient convaincus du secours que leur avaient apporté les Dioscures par leur assistance personnelle. Certains avaient vu de leurs propres yeux les dieux participer au combat; on ne doutait pas que c'étaient les fils de Zeus et que leur aide avait apporté la victoire malgré la supériorité numérique des ennemis. Cette intervention divine avait frappé aussi les esprits des peuples italiques avec une telle force que leur mémoire en restait marquée lorsque, près d'une génération plus tard, à la bataille du lac Régille, les Romains connurent la même aventure. Eux aussi virent deux cavaliers inconnus attaquer irrésistiblement les troupes ennemies et donner la victoire aux Romains. Il s'y ajouta un deuxième miracle : les citoyens restés à Rome ne furent pas informés de la victoire sur les Latins par un message de leur dictateur, mais par le récit de deux cavaliers qui abreuvaient leurs chevaux à la fontaine de Juturne, les mêmes sans doute que ceux qui avaient combattu au lac Régille. Ces messagers inconnus disparurent avant que les esprits excités par cette nouvelle inattendue aient retrouvé leur calme. Le miracle de la bataille de la Sagra permit d'espérer que les mêmes divinités interviendraient aussi dans l'avenir de Rome. On ignore si avant de s'engager à dédier un temple aux dieux de Sagra en échange de leur aide, Postumius était convaincu de l'efficacité de son vœu - un doute eût été humainement compréhensible -, mais il ne voulut pas écarter la possibilité d'un secours, même copié sur un exemple étranger. Il espéra, on peut le penser, que le miracle locrien se renouvellerait; dans ces conditions, les Romains pouvaient s'en inspirer. À Rome, on tolérait parfaitement la romanisation des dieux étrangers avec un culte à l'intérieur même du pomerium, la limite rituelle de la ville ${ }^{64}$. Le respect de l'engagement se traduisait par des frais énormes dépensés pour la construction du temple situé sur le forum Romanum et l'érection des images des dieux devant le sanctuaire. Les Romains l'appelaient aedes

64 Sans la coopération des IIviri s.f., sacerdoce compétent pour l'admission des divinités étrangères au culte romain. 
Castoris, signe de leur aversion envers l'idée d'une parenté entre les divinités.

Les Romains, comme je l'ai déjà décrit, firent également la connaissance du dieu grec Apollon avant les Étrusques, auxquels ils transmirent son nom sous la forme déjà latinisée apulu. Cela permet d'imaginer que l'on avait dès le Ve siècle av. J.-C., attribué une signification affectueuses à Apollon dont la qualité correspondait à un besoin des Romains. Mais puisqu'il n'était pas encore mentionné dans les indigitamenta Pompiliana, c'est-à-dire dans les instructions religieuses de Numa Pompilius, ni dans le calendrier du VIe siècle, on ne doit dater son arrivée à Rome que dans les dernières années du VIe siècle ou un peu plus tard, probablement à l'époque où Sostrate l'Éginate mentionné par Hérodote ${ }^{65}$ voyageait le long des côtes de l'Italie et érigeait un monument en l'honneur d'Apollon sur la plage de Graviscae, aujourd'hui Porto Clementino ${ }^{66}$.

L'Apollon apporté par les commerçants grecs n'était pas le dieu brillant de l'Olympe, comme le présentent les poètes de l'époque d'Auguste, mais selon l'opinion de Karl Kerényl ${ }^{67}$ avec lequel je suis d'accord sur ce point, il était une divinité que l'on craignait. Son pouvoir était formidable et cruel; d'après l'hymne homérique, les autres dieux de l'Olympe avaient peur de sa puissance. À Rome, on croyait le reconnaître dans l'image du Véiovis 68 capitolin portant des flèches mortelles. Son caractère était ambivalent : comme Tortor ${ }^{69}$, il tourmentait les hommes, et comme $A_{p e r t a}{ }^{70}$, à mon avis une forme latinisée du grec apeirktes «le défenseur", "celui qui détourne", il les défendait contre les maladies. Ainsi les Romains exécutèrent dans son sanctuaire des prés Flaminiens une obsécration en 436 av. J.-C. ${ }^{71}$ pour bannir une peste, obsécration signifiant une action magique devant une divinité qui répand l'effroi et dont la nature ne s'est pas encore vérifiée comme favorable ou nuisible. L'obsécration force le dieu à accorder sa bienveillance aux hommes. Il était risqué, à cette époque reculée, de s'adresser à un dieu qui n'avait perdu ni son nom grec ni son image

65 HDT., IV, 125.

66 S. MoscaTr, Italia sconosciuta, p. 138 sq.

67 K. KÉRENYI, Apollon 2 , p. 44.

68 AULU GELLE, V, 12, p. 11 sq.

69 SuÉtone, Auguste, 70, 2.

70 PAul. Festus, 21, p. 1 sq. Lindsay.

71 TtTe Live, IV, 21, 5. 
étrangère. Mais cette mesure magique ou déjà cultuelle eut un résultat heureux.

\section{Latinisation des cultes grecs}

Les dieux grecs arrivés à Rome au VIe ou au Ve siècle av. J.-C. gardèrent leurs noms, tandis que, au IIIe siècle, la diffusion de la mythologie grecque introduisit de nouvelles habitudes et un bouleversement dans la manière de s'exprimer. Les poètes de langue latine, tous sans exception d'origine non romaine, cherchaient des noms latins pour rendre intelligibles aux Romains les dieux et les héros grecs. Quelquefois ce furent des identifications comme Jupiter pour Zeus et Junon pour Héra, Diane et Minerve pour Artémis et Athéna, Neptune et Saturne pour Poseidon et Kronos, sans garder l'accord correct et sans correspondre aux fonctions originelles; quelquefois on inventa des noms qui semblaient être une sorte de traduction comme Dispater pour Plouton, Moneta pour Mnemosyne et Morta pour Moira. Par cette voie, les rapports de parenté connus de la mythologie grecque entrèrent aussi dans la poésie latine de Livius Andronicus, de Névius et de Plaute, mais, comme je l'ai démontré par l'exemple du culte de la Fortuna publica populi Romani Quiritium Primigenia, ils restèrent en dehors du culte officiel et ne pénétrèrent pas dans la pensée religieuse du peuple, du moins à l'époque républicaine, ni dans les familles de la noblesse.

Quand l'esclave Alcésimarque, dans la Cistellaria ${ }^{72}$ de Plaute, sème un désordre épouvantable dans la parenté mythique de Jupiter, Junon, Ops et Saturne, la maladresse amuse les auditeurs, mais elle ne les blesse pas dans leur sentiment religieux. Ennius récite la liste des douze grandes divinités grecques qui ont été honorées à l'occasion du lectisterne ${ }^{73}$ de 217 av. J.-C., lorsque celles-ci remplacèrent pour la première fois les divinités romaines. Le culte romain ne fut pas touché par ces produits de la littérature; la nomenclature grecque et l'imagination divine furent simplement latinisées. La conséquence en fut une identification erronée, qui obscurcit la connaissance des divinités originairement romaines. Seuls les pénates et les lares surent garder leur particularité originelle romaine, parce qu'ils ne se

72 PlaUte, Cistellaria, p. 512 sq.

73 Au lectisterne de 399 av. J.-C. furent réunis Apollo et Latona, Diana et Hercules, Mercurius et Neptunus (TITE Live, V, 13, 6; DeNYs HAL., Ant. rom., 12, 9); les participants du lectisterne de 217 av. J.-C. (TIte LIVE, XXII, 10, 9) correspondent aux dieux grecs de ENNIUS, Annales, p. 62 sq. 
laissaient pas ranger dans le monde fantastique de la mythologie grecque.

Quand je parle d'une latinisation de la mythologie grecque, c'est un point de vue tout à fait nouveau et important. L'idée de la prépondérance de l'influence grecque sur les Romains s'en trouve modifiée; la situation nouvelle correspond à la manière dont les Romains toléraient l'étranger chez eux et l'acceptaient en l'incorporant dans leur mode de vie sans modifier leurs coutumes héritées. Ils assimilaient et s'appropriaient si profondément l'étranger que celui-ci passait finalement pour romain.

\section{Les fềtes}

Un trait caractéristique de la vie religieuse des Romains apparaît dans les manifestations cultuelles collectives. Le culte des dieux à Rome connaît plus d'une quarantaine de fêtes par an, qui non seulement offrent des jours fériés, mais célèbrent des événements auxquels la population participe en général. On peut trouver plusieurs raisons à cette pratique communautaire : tout d'abord, pendant les fêtes, on exécute des actes quasi magiques en quête de lustratio, de la purification des gens dans une sorte de soulagement de la conscience; puis on cherche à se protéger par un acte apotropaïque contre toute menace immatérielle; enfin, on demande par la bénédiction la promesse d'un avenir heureux, garanti par l'aide des dieux. Dans ce processus, tous les membres de la communauté gagnent le sentiment de ne plus être isolés, mais d'être réunis devant la divinité. Ensuite le service des dieux est l'occasion d'une rencontre, d'un acte à la fois social et religieux. Enfin, troisièmement, une motivation très banale, mais importante, réunit les individus devant les autels des dieux : la distribution et la réception de la viande provenant des sacrifices.

Ainsi la réalité de la vie humaine, avec ses obligations naturelles, se soumet à l'idée religieuse. Le culte des dieux se fonde sur la conviction des Romains de ne pouvoir exister sans l'observation correcte du contrat divin que le populus a ratifié et dont la mentalité religieuse spécifiquement romaine présume toujours l'existence. Une explication non simplifiée, mais très simple déjà de son origine établie sur les nécessités fondamentales de la vie romaine.

Marienburger Allee 50

Gerhard RADKE

D - 1000 BERLIN 19 